\title{
Dual Cytomegalovirus and Aspergillus Pneumonia Following In- fluenza B Infection in a Patient with Polyarteritis Nodosa
}

\author{
Chiu-Yin Yeh ${ }^{1}$, Hsi-Hsing Yang ${ }^{1,2}$ and Wen-Liang $\mathrm{Yu}^{1,3 *}$ \\ ${ }^{1}$ Department of Intensive Care Medicine, Chi Mei Medical Center, Tainan, Taiwan \\ ${ }^{2}$ Department of Biotechnology, Southern Taiwan University of Science and Technology, Tainan, Taiwan \\ ${ }^{3}$ Department of Medicine, School of Medicine, College of Medicine, Taipei Medical University, Taipei, Taiwan
}

*Corresponding author: Wen-Liang Yu, Department of Intensive Care Medicine, Chi Mei Medical Center, Department of Medicine, School of Medicine, College of Medicine, Taipei Medical University, 901 Chung Hwa Rd., 710 Tainan City, Taiwan, Tel: +886-6-2812811, Fax: +886-6-2833351,_E-mail:Yuleon_md@yahoo.com.tw

\begin{abstract}
Polyarteritis Nodosa (PAN) is a systemic necrotizing vasculitis that might require immunosuppressive therapy. We report on a 72-year-old woman of PAN with influenza B infection, who developed dual cytomegalovirus and Aspergillus pneumonia in the later course. Together with steroid therapy, delayed initiation of anti-CMV and anti-fungal therapy complicated the disease course. Early diagnosis and therapy for CMV and aspergillosis in the patients post-influenza attack are important, especially in those with steroid therapy.
\end{abstract}

\section{Keywords}

Aspergillosis, Cytomegalovirus, Influenza B, Polyarteritis nodosa

\section{Introduction}

Polyarteritis Nodosa (PAN) is a form of systemic necrotizing vasculitis preferentially targeting medium muscular arteries. The clinical responses to immunosuppressive therapy support the pathogenic linkage of PAN to immunological mechanisms [1]. Cytomegalovirus (CMV) diseases frequently occur in the patients receiving immunosuppressive therapy. In addition, Invasive Pulmonary Aspergillosis (IPA) usually occurs in immunocompromised hosts, but can also happen after severe influenza infections in patients without classical predisposing factors [2]. Herein we report on a patient of PNA with influenza B infection, who subsequently developed life-threatening dual CMV and Aspergillus pneumonia.

\section{Case Report}

A 72-years-old woman of polyarteritis nodosa had fever, running nose, and nasal obstruction for 3 days. In emergency room, rapid influenza diagnostic test in nasopharyngeal swab was positive for influenza B on October 31, 2015. Chest X-ray (CXR) showed slight infiltration on bilateral lungs. A white blood cell count was $16,100 / \mu \mathrm{L}$ and an elevated C-Reactive Protein (CRP) level was $238.0 \mathrm{mg} / \mathrm{L}$. Initial renal function was normal. Other data of laboratory testing were tabulated in Table 1.

Initially, zanamivir inhalation therapy and intravenous moxifloxacin were given. However, high spiking fever was still off and on. As increased CRP (315.7 mg/L), leukocytosis $(24,500 / \mu \mathrm{L})$ and worsening dyspnea, the patient was transferred to the intensive care unit on November 09. Bilevel positive airway pressure was used for non-invasive mechanical ventilation support. Antimicrobial therapy with piperacillin-tazobactam was initiated to replace moxifloxacin. Thereafter, fever was subsided. As acute renal failure occurred, continuous venovenous hemofiltration was performed.

Followed-up CXR showed newly developed consolidation over right lung fields (Figure $1 \mathrm{~A}$ ) and the sputum cultures yielded yeast-like organisms. Antimicrobial therapy was maintained with piperacillin-tazobactam. For the therapy of PAN, intravenous methylprednisolone 40 mg every 6 hours was given since November 20,

Citation: Chiu-Yin Y, Hsi-Hsing Y, Wen-Liang Y (2017) Dual Cytomegalovirus and Aspergillus Pneumonia Following Influenza B Infection in a Patient with Polyarteritis Nodosa. Int J Virol AIDS 3:034. doi. org/10.23937/2469-567X/1510034

Received: July 31, 2017: Accepted: September 18, 2017: Published: September 20, 2017

Copyright: (C) 2017 Chiu-Yin Y, et al. This is an open-access article distributed under the terms of the Creative Commons Attribution License, which permits unrestricted use, distribution, and reproduction in any medium, provided the original author and source are credited. 
Table 1: Clinical course and laboratory data of the patient during hospitalization.

\begin{tabular}{|c|c|c|c|c|c|c|c|c|c|c|}
\hline Laboratory data (normal limits) & Oct 31 & Nov 3 & Nov 6 & Nov 9 & Nov 16 & Nov 20 & Nov 25 & Nov 30 & Dec 3 & Dec 10 \\
\hline Admission & Ward & Ward & Ward & ICU & ICU & ICU & ICU & ICU & ICU & ICU \\
\hline Temperature (peak, $\left.{ }^{\circ} \mathrm{C}\right)$ & 39.2 & 40.4 & 39.2 & 37.8 & 37.2 & 36.8 & 36.8 & 36.8 & 36.8 & 36.0 \\
\hline Antibiotic therapy & A & B & B & C & C & C & $\mathrm{D}$ & $\mathrm{D}$ & $\mathrm{D}$ & $\mathrm{D}$ \\
\hline Influenza $A$ antigen & Negative & & & & & & & & & \\
\hline Influenza B antigen & Positive & & & & & & & & & \\
\hline Urine RBC/HPF & $30-49$ & $30-49$ & & $20-29$ & & & & & & \\
\hline Urine WBC/HPF & $10-19$ & $30-49$ & & $30-49$ & & & & & & \\
\hline Urine culture & NG & NG & & NG & & & & & & \\
\hline Blood culture & NG & NG & NG & & & & & & & \\
\hline Sputum culture & & & & & Yeast & & & & & \\
\hline Blood WBC count/ $\mu \mathrm{L}$ & 16,100 & 14,500 & 19,600 & 24,500 & 34,500 & 31,100 & 18,100 & 17,300 & 19,200 & 9,000 \\
\hline Blood platelet count/ $\mu \mathrm{L}$ & 449,000 & 435,000 & 495,000 & 600,000 & 214,000 & 173,000 & 99,000 & 89,000 & 41,000 & 55,000 \\
\hline Blood hemogram (g/dL) & 12.4 & 10.8 & 9.9 & 8.1 & 6.9 & 6.1 & 8.6 & 8.8 & 8.8 & 9.3 \\
\hline $\mathrm{CRP}(<5 \mathrm{mg} / \mathrm{L})$ & 238.0 & 319.1 & 296.0 & 315.7 & 183.6 & 175.0 & 34.7 & 22.8 & 72.6 & 62.7 \\
\hline Procalcitonin $(<0.05 \mathrm{ng} / \mathrm{mL})$ & & & & 7.23 & & 24.22 & & & 1.14 & \\
\hline BUN $(6-20 \mathrm{mg} / \mathrm{dL})$ & & 13 & & 61 & 117 & 63 & 54 & 162 & 43 & 93 \\
\hline Creatinine $(0.57-1.11 \mathrm{mg} / \mathrm{dL})$ & 0.77 & 0.82 & & 4.52 & 8.72 & 4.01 & 1.46 & 5.09 & 1.28 & 2.16 \\
\hline AST (5-34 U/L) & 34 & & & & & & & & & 53 \\
\hline ALT (2-40 U/L) & 37 & & & & & & & & & 69 \\
\hline CMV-PCR & & & & & & Positive & & Positive & & \\
\hline Aspergillus $\mathrm{Ag}(<0.5$ index) & & & & & & & 0.32 & & & 6.37 \\
\hline
\end{tabular}

ICU: Intensive Care Unit; Antibiotic A: Cefuroxime (for 4 days); Antibiotic B: Moxifloxacin (for 5 days); Antibiotic C: Piperacillintazobactam (for 16 days); Antibiotic D: Piperacillin (for 18 days); RBC: Red Blood Cell; WBC: White Blood Cell; HPF: High Power Field; NG: No Growth; CRP: C-Reactive Protein; BUN: Blood Urea Nitrogen; AST: Aspartate Aminotransferase; ALT: Alanine Aminotransferase; CMV-PCR: Cytomegalovirus-Polymerase Chain Reaction.

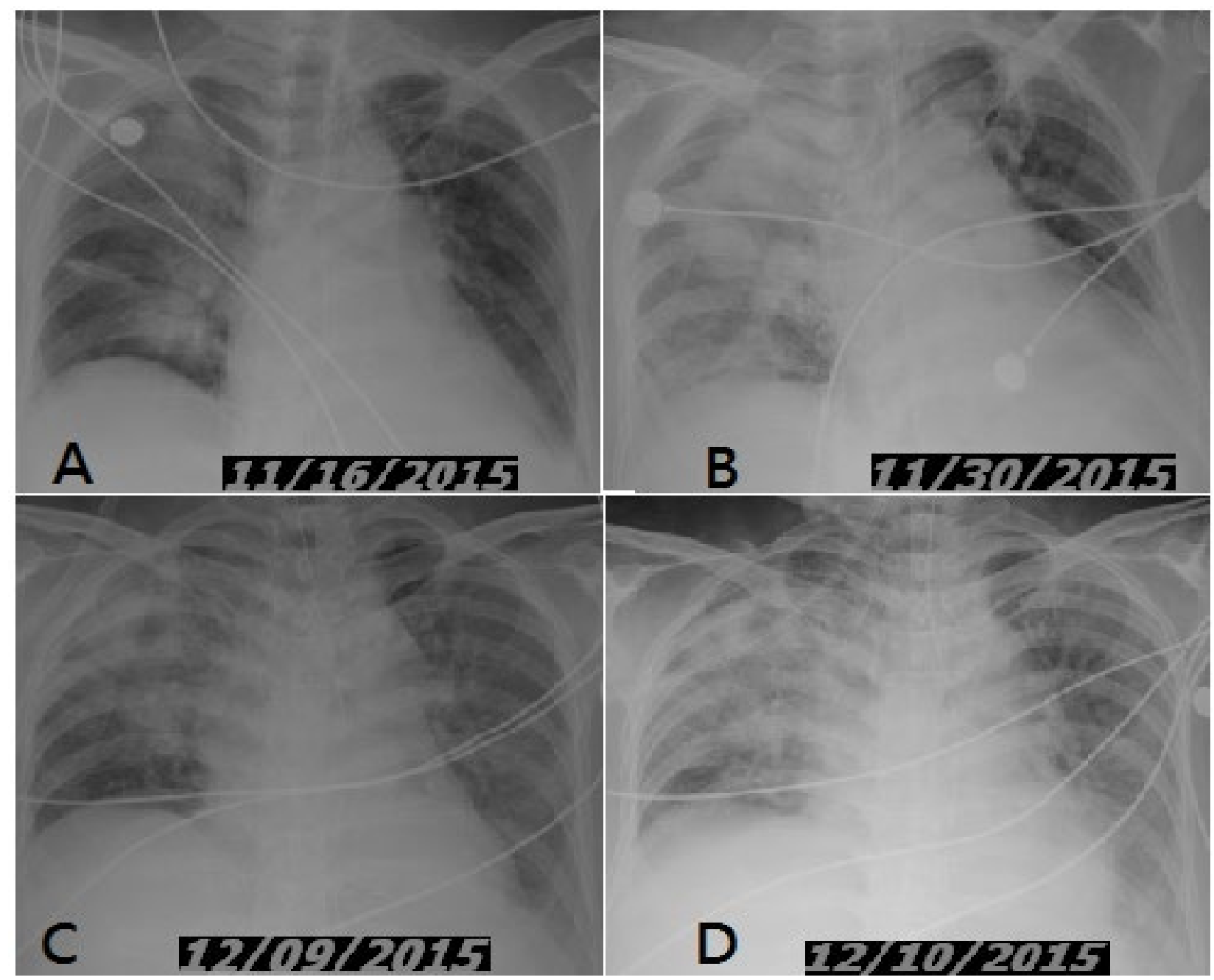

Figure 1: A) CXR is showing consolidation in right lung fields; B) Worsening in right upper lung field; C) Progress in cavity formation; D) Into more extensive consolidation. 
which had been maintained for more than 3 weeks until death. Meanwhile, blood CMV-DNA testing by Polymerase Chain Reaction (PCR) showed positive. The serum Aspergillus antigen index revealed 0.32 (normal, < 0.5) on November 25, 2015. However, the CXR showed extended consolidation over right upper lung field on November 30 (Figure 1B). Followed-up blood CMV-PCR testing remained positive and thus ganciclovir was given since December 01. Nonetheless, CMV antigenemia revealed 8 positive cells per 200,000 leukocytes on December 08 . The CXR revealed cavity formation over right upper lung field (Figure 1C) with progression to more extensive consolidation (Figure 1D). Family decided comfort therapy for the patient. The serum Aspergillus antigen index was elevated to 6.37 on December 10, 2015. The patient passed away on December 12, 2015. Anti-fungal therapy was not given in time.

\section{Discussion}

The current patient with PAN developed dual CMV and IPA following influenza B infection. While pneumonia was worsening, only blood CMV DNA but not Aspergillus antigen was detectable, it could indicate development of CMV pneumonia. However, initiation of antiviral therapy was hesitated. The occurrence of CMV infection due to the use of corticosteroid or immunosuppressive therapy for PAN is often life-threatening, so prompt intravenous antiviral treatment is recommended [3]. Oral cyclophosphamide and prednisone are standard treatment for PAN. However, Mayer, et al. referred to the effectiveness of less aggressive treatment without immunosuppressants for immunogenic vasculitis concurrent with CMV infection [4]. Because our patient had no proteinuria, renal damage (initially), cardiomyopathy, gastrointestinal manifestations, and/or central nervous system involvement, she was also treated with corticosteroid alone without immunosuppressants, which led to partial improvement of clinical condition, such as decreasing inflammatory markers (leukocyte count, CRP and procalcitonin). Nonetheless, CMV pneumonia and subsequent identified IPA still complicated the disease course, even though aggressive cytotoxic treatment was not given for PAN.

It should be noticed that post-influenza A status has been recognized as a risk host for IPA in many countries [2]. Post-influenza IPA could be fatal, even on aggressively intensive care and management $[5,6]$. Besides, we recently reported a 76-year-old patient with influenza B pneumonia coexisting with IPA [7]. Although rarely reported, influenza $B$ might predispose to IPA in our patient with PAN on steroid therapy. Therefore, several factors including influenza B, PAN, steroid use, CMV infection and aspergillosis could all contribute to a lethal disease in our patient. Early diagnosis and treatment of CMV and aspergillus infection is of the utmost importance to avoid morbidity and mortality. The use of piperacillin-tazobactam or piperacillin before Asper- gillus antigen testing should not be a concern for false positivity in our institute [8].

In conclusion, we report a patient of PAN who developed dual CMV and Aspergillus pneumonia following the influenza $B$ infection. Physicians should be highly alert to the unusual occurrence. Repeated laboratory tests could not be neglect. Our case highlights the CMV and Aspergillus dual infection as the possible complication of influenza B in a patient treated with steroid therapy. Early diagnosis and therapy for suspected CMV and Aspergillus infections are indeed important.

\section{Acknowledgement}

None declare.

\section{Conflict of Interests}

The authors declare that they have no conflict of interest and no financial support regarding this work. The above study has been granted exemption from review by the Institutional Review Board of Chi-Mei Medical Center (application no.10501-E01).

\section{References}

1. Sellar RJ, Mackay IG, Buist TA (1986) The incidence of microaneurysm in polyarteritis nodosa. Cardiovasc Intervent Radiol 9: 123-126.

2. Crum-Cianflone NF (2016) Invasive aspergillosis associated with severe influenza infections. Open Forum Infect Dis 3: 171.

3. Sunnetcioglu A, Sunnetcioglu M, Emre H, Soyoral L, Goktas U (2016) Cytomegalovirus pneumonia and pulmonary haemorrhage in a patient with polyarteritis nodosa. J Pak Med Assoc 66: 1484-1486.

4. Meyer MF, Hellmich B, Kotterba S, Schatz H (2000) Cytomegalovirus infection in systemic necrotizing vasculitis: causative agent or opportunistic infection? Rheumatol Int 20: 35-38.

5. Ku YH, Chuang YC, Yu WL (2017) Postinfluenza A (H3N2) refractory invasive pulmonary aspergillosis. J Formos Med Assoc 116: 404-405.

6. Su BA, Yu WL (2017) Failure of extracorporeal membrane oxygenation to rescue acute respiratory distress syndrome caused by dual infection of influenza $A(\mathrm{H} 1 \mathrm{~N} 1)$ and invasive pulmonary aspergillosis. J Formos Med Assoc 116: 563564.

7. Su MY, Tan CK, Yang CC, Yu WL (2016) Invasive pulmonary aspergillosis following influenza: Report of two cases. Open J Clin Med Case Rep 2: 1-6.

8. $\mathrm{Ku} \mathrm{YH}$, Chan KS, Yang CC, Tan CK, Chuang YC, et al. (2017) Higher mortality of severe influenza patients with probable aspergillosis than those with and without other coinfections. J Formos Med Assoc 116: 660-670. 